\title{
"La géométrie du pouvoir ». Peuples indigènes et révolution au Venezuela
}

\section{Alexander Mansutti Rodriguez et Catherine Alès}

\section{Q OpenEdition}

\section{Journals}

Édition électronique

URL : https://journals.openedition.org/jsa/8293

DOI : $10.4000 /$ jsa.8293

ISSN : 1957-7842

Éditeur

Société des américanistes

\section{Édition imprimée}

Date de publication : 2 décembre 2007

Pagination : 173-193

ISSN : 0037-9174

\section{Référence électronique}

Alexander Mansutti Rodriguez et Catherine Alès, « «La géométrie du pouvoir ». Peuples indigènes et révolution au Venezuela », Journal de la Société des américanistes [En ligne], 93-2 | 2007, mis en ligne le 05 février 2008, consulté le 04 septembre 2022. URL : http://journals.openedition.org/jsa/8293 ; DOI : https://doi.org/10.4000/jsa.8293 


\title{
CHRONIQUE DU GROUPE D'INFORMATION SUR LES AMÉRINDIENS
}

\author{
VENEZUELA
}

\section{« LA GÉOMÉTRIE DU POUVOIR ». PEUPLES INDIGÈNES ET RÉVOLUTION AU VENEZUELA}

Les indigènes ont joué un rôle fondamental dans le processus révolutionnaire guidé par Hugo Chávez au Venezuela depuis décembre 1998. Ils sont entrés dans cette période de changement avec de longues années de lutte derrière eux et un agenda propre devant être négocié dans le cadre du bloc hétérogène qui a mené Chávez au pouvoir. Presque neuf années après avoir établi cette alliance, force est de constater que le mouvement indigène a subordonné ses objectifs idéaux aux exigences du processus révolutionnaire mis en place par le " chavisme », lequel se révèle chaque jour davantage un processus centralisateur, attaché à concentrer le pouvoir et reposant sur la figure d'un leader puissant.

Aujourd'hui, l'alliance entre le chavisme et les peuples indigènes se maintient, mais sous une forme flexible qui se rappoche du socialisme bolivarien. La révolution vénézuélienne a avancé, non sans diffïcultés. Jusqu'au coup d'État du mois d'avril 2002, Chávez est parvenu à négocier institutionnellement ses initiatives dans le cadre du jeu démocratique. Puis, une fois qu'il a réussi à sortir des factions qui lui étaient hostiles à l'intérieur de la "Force Armée " ${ }^{1}$ et que la défaite de la grève générale qui s'est déroulée de décembre 2002 à février 2003 lui a permis de prendre le contrôle absolu de la compagnie pétrolière nationale (PDVSA) et de briser la résistance organisée par le patronat et les syndicats traditionnels, le processus révolutionnaire est accéléré et dirigé vers une concentration croissante du pouvoir au sein de l'exécutif national. Les formes démocratiques conventionnelles ont été affaiblies pour faciliter la transition révolutionnaire. Dorénavant, le chavisme peut dire ouvertement que son projet est socialiste. Certes, il le rapporte au $\mathrm{xxI}^{\mathrm{e}}$ siècle afin de le différencier du communisme soviétique, mais il conserve quelques éléments fondamentaux du socialisme traditionnel comme l'antagonisme entre capitalisme et socialisme, la lutte des classes en tant que moteur des changements et la nécessité de modeler un « homme nouveau ». Malgré l'effort pour maintenir les procédures démocratiques telles que les 
élections et se distinguer idéologiquement des formes autoritaires de socialisme, il est évident que le chavisme contrôle ouvertement tous les pouvoirs de l'État dans un pays où il existe cinq pouvoirs séparés, théoriquement autonomes, le pouvoir exécutif, contrôlé par le président élu, le pouvoir législatif, le pouvoir judiciaire, le pouvoir électoral et le pouvoir citoyen ou moral. Il s'agit, par conséquent, d'un pouvoir formellement réparti en cinq domaines appelés à s'équilibrer entre eux, mais, ici, contrôlés par la volonté d'une seule personne, celle du chef de l'État. Cet exercice du pouvoir s'est vu légitimé consécutivement par les élections nationales, régionales et locales gagnées par le chavisme à l'aide d'une organisation électorale entraînée par l'organisation militaire. D'un point de vue politique et administratif, cette situation de resserrement des pouvoirs, légalement autonomes, autour du seul chef de l'État commence à s'exprimer à partir du triomphe de Chávez au referendum révocatoire du 15 octobre 2004. Des politiques publiques tendent à annuler tous les efforts antérieurs de régionalisation et de décentralisation de l'État et, simultanément, à lier directement les forces organisées des communautés locales avec la présidence de la République. Les « conseils communaux » qui sont créés par la loi du 10 avril 2006 sont l'expression la plus achevée de cette politique (RBV 2006a). Il s'agit d'organisations communautaires qui dépendent directement du secrétariat de la présidence de la République. Elles ont pour but de développer les principes de la démocratie participative et " protagoniste ${ }^{2}$ dans la gestion des ressources financières pour l'exécution de projets de développement local qui, jusque-là, étaient sous la responsabilité des mairies et des gouvernements régionaux ${ }^{3}$. Avec cette politique de fragmentation et de fragilisation des pouvoirs et des institutions qui ne relèvent pas du gouvernement central, les intermédiaires traditionnels, les maires et gouverneurs d'État élus qui disposent d'un pouvoir majeur face à la présidence de la République, s'aftaiblissent. Le pouvoir présidentiel se renforce, tandis que le pouvoir de médiation est morcelé entre de multiples petites communautés locales. Ainsi reconfigurée, la centralisation conduit à fortifier les pouvoirs de l'État et à mettre directement ce dernier en relation avec les pouvoirs, limités, des localités.

Le président Chávez apparaît comme un homme charismatique, doué d'une facilité de communication avec les secteurs populaires. Son discours se nourrit des héros et des mythes fondateurs de la nationalité vénézuélienne. Des grands hommes de la nation comme Simón Bolívar, l'éducateur Simón Rodríguez et le Général des hommes libres, Ezequiel Zamora, meneur des factions fédéralistes qui se lancèrent contre les latifundistes au milieu du XIX ${ }^{\mathrm{e}}$ siècle, sont convoqués sans répit. Dans ce contexte de revendication des grands idéaux qui ont présidé à l'édification du Venezuela, les Indiens occupent une place de choix. Dans le préambule de la constitution de 1999, les « aborigènes » sont placés au premier rang, aussi bien reconnus dans le présent, en inscrivant le Venezuela comme une nation "pluriculturelle et multiethnique ", que magnifié dans les origines, en qualifiant leur geste d'hérö̈que et de sacrificielle : 
El pueblo de Venezuela en ejercicio de sus poderes creadores e invocando la protección de Dios... y el heroismo y sacrificio de nuestros antepasados aborígenes y de los precursores y forjadores de una patria libre y soberana... (RBV 2000)

De même, l'indigène va être très présent dans le discours de la révolution. Quand on évoque la force et le caractère, Guaicaipuro, l'un des chefs de la résistance indigène, est remémoré. Quand on invoque le socialisme, les indigènes sont posés comme les maîtres en la matière. Quand on retrace la libération latino-américaine, les peuples indigènes sont exposés en première ligne. Les discours du Président mentionnent les indigènes de façon itérative, et des leaders indigènes l'accompagnent dans ses tournées latino-américaines.

Cependant cette politique ne va pas sans carences ni contradictions. Comme nous le verrons plus loin, la légitimation des droits territoriaux est en berne, ceux-ci étant déniés lorsqu'il s'agit de reconnaître les grands territoires. L'État esquive régulièrement le droit à la consultation préliminaire dans le cas des macro-projets de gazoducs que celui-ci met en œuvre dans la Guajira avec la Colombie et pour le MERCOSUR dans la Guayana. Face à cela, le mouvement indigène garde le silence.

Le fait est que le mouvement chaviste s'est transformé. Il a débuté à partir d'une alliance composite comprenant, outre les indigènes, des groupes d'exclus de l'économie formelle comme les vendeurs des rues (buhoneros) et des groupes alternatifs périphériques (tels que les homosexuels, les artistes, les féministes, les environnementalistes, les partisans de l'avortement, les défenseurs des minorités culturelles, religieuses et sexuelles), et sans l'appui des groupes institutionnalisés comme les syndicats et les corporations professionnelles. Aujourd'hui, il se déplace vers des secteurs plus organisés comme les cercles bolivariens, les syndicats parallèles créés par le gouvernement, les organisations de base comme les conseils communaux et les partis politiques émergents du chavisme (PPT, MVR, parti communiste, PUAMA) auxquels Chávez a demandé, non sans résistance de la part des intéressés, de se dissoudre au bénéfice du nouveau parti unitaire de la révolution. Il semblerait que, d'une phase séminale fondée sur la ferveur désorganisée des exclus, on passe à une phase qui s'étaye sur des secteurs structurés qui incluent des ouvriers et des intellectuels, tous intégrés au sein d'un parti représentant l'avant-garde de la révolution.

\section{LE MOUVEMENT INDIGÈNE AU MOMENT DE L'AVÈNEMENT DU CHAVISME}

Ce sont les processus même d'intégration et de changement, ayant pris place au Venezuela avant l'arrivée du chavisme au pouvoir, qui ont créé les conditions rendant possible son succès auprès des indigènes. Même si l'expérience d'articulation à la société nationale des peuples indigènes vénézuéliens est loin d'être en tout lieu identique tant par sa profondeur que sa massivité ${ }^{4}$, l'on peut tout à fait 
dire que, pour chacun d'entre eux, l'action combinée, quoique inégale, de la christianisation, de la scolarisation, des actions sanitaires, de l'intégration au marché économique, des partis et des structures étatiques de proximité (comme les préfectures et les municipalités) a ouvert les espaces d'échange culturel qui ont facilité la consolidation de relations de dépendance et de subordination des indigènes face à l'État.

Comme élément de première importance, pour son impact récent, on peut inscrire la municipalisation des régions à population indigène qui, comme nous l'avons montré par ailleurs, a fortement perturbé la quotidienneté de la vie autochtone. Dans l'État Amazonas, par exemple, où cette institution étatique n'existait que pour la ville de Puerto Ayacucho, la soudaine création de sept municipalités en territoire indigène a engendré force conflits et séparations au sein des familles et des communautés, largement à inscrire au crédit de la pression exercée par les partis politiques et l'introduction de formes de pouvoir exogènes au monde indigène (Alès 2003 ; 2007). En Amazonas, les organisations indigènes s'opposèrent à l'imposition du modèle créole de municipalisation, mais, pour la première fois également, des indigènes, comme Jaime Turón et Bernabé Arana, soutenus par les partis gagnèrent des élections pour devenir les maires de municipalités. D'autres furent élus comme conseillers et commencèrent à participer à la gestion des budgets publics destinés aux communautés indigènes. Régionalement, une fois perdue la lutte pour défaire la division politico-territoriale telle qu'elle était imposée par les partis, plusieurs leaders des organisations indigènes se sont portés candidats pour obtenir des postes d'élus dans les municipalités.

Les organisations et leurs dirigeants participèrent également de plus en plus au mouvement indigène aux niveaux national et international. De telle façon que, lorsque Chávez gagna les élections, les organisations indigènes vénézuéliennes disposaient d'un groupe de dirigeants expérimentés, de premier rang ou intermédiaires, qui connaissaient relativement le fonctionnement du système politique créole et savaient y naviguer. Elles avaient en outre un programme indigène, mûri au long des années de combat, qui comprenait comme points fondamentaux la reconnaissance des territoires indigènes, la revalorisation des cultures autochtones et de sa résistance face à la colonisation, et la représentation des aborigènes dans les organes législatifs de l'État. Quelques-uns de leurs plus illustres dirigeants intervenaient dans les institutions du monde, non sans habileté, quand nombre de leurs homologues créoles commençaient à peine à se familiariser avec ce dernier. Noelí Pocaterra est parvenue à être vice-présidente du Conseil mondial des Peuples Indigènes de l'ONU, et Jose Luis González à devenir président de l'Alliance pour le climat, une organisation réunissant plus de trois cents maires européens apportant leur soutien aux luttes des peuples indigènes (Mansutti Rodríguez 1992). En outre, les organisations indigènes possédaient une structure nationale représentative, le Conseil national indien du Venezuela (CONIVE). 


\section{La nouvelle Constitution, la légalité émergente et les législateurs INDIGÈNES}

La consolidation du bloc social qui mène Hugo Chávez au pouvoir en décembre 1998 bénéficie de l'appui enthousiaste des organisations indigènes vénézuéliennes. Ces dernières se réunissent avec lui, au mois de mars de l'année suivante, afin de confirmer son engagement de promouvoir leurs revendications les plus sensibles une fois qu'il aura gagné les élections. En août 1999, lorsque s'installe l'Assemblée nationale constituante, cette alliance a déjà porté ses fruits avec la présence de trois constituants indigènes - élus en assemblée par les seuls indigènes sous la supervision de l'organisme électoral vénézuélien - pour représenter dans cette instance leurs intérêts (Mansutti Rodríguez 2000 ; Alès 2007). Dans le travail réalisé par les membres indigènes de l'Assemblée nationale constituante dans le cadre de l'alliance avec le chavisme, on note que, dans la nouvelle Constitution, sont inclus dix-huit articles et trois dispositions transitoires qui mentionnent expressément les indigènes.

La nouvelle Constitution contient deux dispositions qui garantissent la présence indigène dans les organes législatifs de la nation et qui sont d'une importance cruciale pour comprendre la vitalité avec laquelle la révolution est allée de l'avant sur le plan légal. L'article 125 établit la garantie de la représentation politique indigène dans les organes délibérants, tant à l'Assemblée nationale que dans les entités législatives régionales et locales à population indigène. Pour sa part, la septième disposition transitoire établit que les députés en représentation à l'Assemblée nationale doivent être au nombre de trois, chacun représentant respectivement une des trois grandes régions du pays où les indigènes sont présents, l'Orient, le Sud et l'Occident. La même disposition établit que ces représentants doivent être élus dans des scrutins où tous les citoyens de la circonscription électorale, indigènes et non indigènes, peuvent voter. De cette manière, il y a trois représentants indigènes à l'Assemblée nationale, élus respectivement par l'ensemble des électeurs de chacune des trois grandes régions, auxquels s'ajoutent un représentant indigène dans les conseils législatifs des neufs États (Zulia, Mérida, Trujillo, Apure, Monagas, Anzoátegui, Sucre, Delta et Amazonas) à population indigène ainsi qu'un représentant dans les conseils municipaux de ces États. Tous sont en position de rédiger des lois et de promouvoir des politiques publiques en faveur des Indiens.

Simultanément, le leadership indigène, qui avait déjà appris à profiter des opportunités offertes par la décentralisation promue par l'ancien régime, s'incorpore et s'adapte aux nouvelles circonstances. En 2000, un politicien d'origine indigène se convertit en gouverneur de l'État Amazonas (Liborio Garulla) et d'autres parviennent à être maires de municipalités dans les États Delta (Antonio Díaz), Zulia (Mara et Paez), Amazonas (Autana, Manapiare et Alto Orinoco) et Bolívar (Gran Sabana). 
L'impact de la participation indigène dans la politique nationale et régionale a été considérable. En effet, cela a créé une infrastructure institutionnelle signalant au leadership indigène une possible carrière politique qui commence avec les conseillers municipaux, passe par les mairies, de là aux conseils législatifs puis à l'Assemblée nationale ou aux gouvernements des États dotés d'une importante population indigène.

Lorsque la nouvelle Assemblée nationale unicamérale, qui se substitue à l'ancien Congrès national, s'installe, les trois députés indigènes réussissent à obtenir la création d'une commission propre, la Comisión Permanente de Pueblos Indígenas. Elle a pour but que s'actualise et aille de l'avant un programme législatif ad hoc qui inclut l'approbation de la résolution 169 de l'OIT et un ensemble de lois qui développe les principes constitutionnels fondamentaux pour les autochtones. À partir de ce moment-là, le programme légal va se réaliser rapidement. En 2001, est approuvé le Convenio 169 de l'OIT (RBV 2001a) - ce qui donne un rang constitutionnel ${ }^{5}$ à ses dispositions comme stipulé dans l'article 23 de la nouvelle Constitution. En 2002, est ratifiée la loi de démarcation et garantie des habitats et terres indigènes (RBV 2001b) et, en 2005, la loi organique des peuples et communautés indigènes (RBV 2005a) dans laquelle est développée une bonne partie des droits reconnus dans la Constitution. En complément à ces lois concernant le monde aborigène, les législateurs indigènes prennent soin que figurent des dispositions se référant aux indigènes dans les autres lois. Citons, entre autres, la loi organique du pouvoir municipal (RBV 2005b), la loi des conseils communaux (RBV 2006a), la loi de la diversité biologique (RBV 2002) et l'éphémère ${ }^{6}$ loi organique pour la planification et gestion du territoire (RBV 2006b).

Simultanément, d'autres mesures politiques sont prises, certes de rang légal mineur, mais qui n'en sont pas moins puissantes symboliquement. Un décret va permettre de porter Guaicaipuro, héroïque résistant indien lors de la conquête espagnole, au panthéon national pour reposer auprès des autres fondateurs de la nation ; des résolutions du ministère de l'Éducation, de la Culture et des Sports oflicialisent les langues autochtones et changent le nom des festivités du 12 octobre, date de l'arrivée de Colomb sur les côtes américaines, qui devient le « Jour de la résistance indigène » (RBV s. d.).

Tandis que les différents points du programme indigène sont en train d'avancer, le degré de conflit politique dans le pays fait que la défense de la révolution, per se, se convertit en un leitmotiv de l'alliance révolutionnaire. Les objectifs indigènes commencent à se diluer et, entre tous, le principal objectif stratégique de la lutte indigène : la démarcation et la remise des titres de propriété de leurs territoires.

Au fur et à mesure que le leadership indigène se compromet avec le pouvoir de l'État, sa présence médiatique et institutionnelle grandit et, en même temps, dans les communautés de base, les critiques qu'elles lui adressent augmentent. Il existe 
actuellement, dans les organisations indigènes, une tension forte entre les communautés et le leadership qui les représente et qui s'implique ouvertement avec les institutions révolutionnaires nationales et étatiques. Du point de vue des communautés, les dirigeants élus étaient appelés à défendre leurs droits et non à bénéficier des prébendes du gouvernement. Il leur est très difficile de comprendre ce que font leurs représentants dans les centres de pouvoir politique et le rôle qu'ils jouent là-bas et, cela, malgré leur retour fréquent dans leur zone d'élection. Les conséquences de l'éloignement des élus avec les communautés sont patentes. En décembre 2005, deux des trois députés indigènes nationaux, tous deux rédacteurs de la nouvelle Constitution, perdirent les élections qui leur auraient permis de renouveler leur charge, l'un au profit d'une dirigeante émergente, l'autre, d'un leader traditionnel, celui qu'il avait battu en 1999 lors des élections pour les constituants indigènes. Les leaders défaits par d'autres leaders indigènes chavistes payaient, outre leurs propres faiblesses, l'inefficacité chronique de l'État vénézuélien à concrétiser ses politiques les plus complexes.

Quel a été le mécanisme de cette évolution ? Lorsque quelques-unes des dispositions constitutionnelles garantissant la présence de l'État dans le monde indigène et l'exercice de droits (tels que la santé, l'éducation et la participation politique) ont commencé à se convertir en politique publique, d'importantes opportunités de travail se sont alors présentées pour le leadership indigène, lesquelles ont été saisies par les indigènes, en petit nombre, mais bien préparés, qui s'occupaient auparavant des petites structures politico-administratives des organisations indigènes. Dans ces dernières travaillaient quelques représentants expérimentés et une génération de relève, petite par sa taille, qui avaient déjà des difficultés pour accomplir les tâches qui leur étaient dévolues, pour beaucoup en raison de leurs faibles moyens financiers. Avec la révolution, les dirigeants ont occupé les postes les plus attractifs et les plus hauts dans la hiérarchie, créés, soit par disposition constitutionnelle, soit par dispositions administratives, et ils ont rempli des fonctions dans le cadre de la révolution bolivarienne. Les fonctions de moindre envergure et de moindre pouvoir réel, de moindre rapport également, furent alors négligées par ceux qui assumaient de nouvelles responsabilités. Elles ont dû être remplies par de nouveaux dirigeants ou par des leaders expérimentés, mais de seconde ligne, sans préparation suffǐsante, lesquels se virent obligés d'apprendre sur le tas.

Dans ce contexte, se sont ouverts des postes de législateurs et de conseillers des législateurs, mais également des charges qui dépendent de l'éducation indigène au ministère, des fonctions dans les directions nationale et régionale de santé indigène, des offices au ministère de l'Environnement, à la défense du Peuple, à l'Institut national des terres, aux Instituts d'attention à l'Indigène, au ministère de la Jeunesse, pour ne citer que les plus importants. Dans les organisations indigènes, il s'est donc produit " un effet d'étirement-essoufflement ", en raison de l'ouverture de postes et du manque de relève en hommes. On entend par cet 
effet ce qui se passe lorsqu'une organisation, à capacité limitée de travail pour mener à bien ses fonctions, se voit subitement obligée de répondre aux tâches associées aux anciennes comme aux nouvelles demandes, et à un moment où ces dernières augmentent brusquement (Mansutti Rodríguez s. d.). Les conséquences de cet effet sont multiples. En premier instance se développe un processus de hiérarchisation des tâches dans la révolution selon leur importance. En seconde instance, cela promeut la négligence des postes qui ne sont pas bien - voire pas du tout - rémunérés, ou qui ne s'intègrent pas dans la ligne des carrières politiques permettant d'arriver aux postes les plus porteurs. En troisième instance, il se produit un effet d'étirement dans les lignes de transmission de l'information entre le dirigeant et sa base politique. Finalement, cela induit une compétition entre les leaders émergents et les dirigeants établis, spécialement de la part des plus jeunes, certains d'entre eux formés à l'université, qui gravissent les échelons à une vitesse jamais vue auparavant (mais que, pour leur part, ils considèrent normale et comme conforme à leurs compétences). Dans ce contexte, un leader indigène, houspillé en interne, soumis à la scrutation permanente de ses idées par l'entourage révolutionnaire, éventuellement menacé de se voir disqualifié et ruiné d'un jour à un autre s'il est perçu par quelque chef de la révolution comme « légèrement contraire à la révolution ", peut seulement maintenir un programme indigène propre tant que celui-ci n'entre pas en conflit avec les projets du président de la République, autrement dit, avec ceux du socialisme du $\mathrm{xxI}^{\mathrm{e}}$ siècle tel qu'il est conçu. Le problème est que, au-delà du symbolique et des services d'assistance, le programme indigène - qui a pour but le renforcement du droit au territoire et à l'autodétermination de modèles de développement adaptés à chaque peuple - entre nécessairement en conflit avec les États modernes. Dans ces conditions, le programme stratégique indigène tend à "s'invisibiliser », tandis que les dirigeants souffrent de la contradiction existant entre leurs compromis avec une alliance politique qui leur a beaucoup offert et les limites qu'impose le gouvernement d'un Etat dans l'accomplissement des objectifs stratégiques indigènes. Les leaders indigènes se retrouvent dans une situation typique de " double bind".

Les communautés, quant à elles, ont été fortement touchées par la révolution, spécialement à partir de 2003 avec l'implantation de ce que le gouvernement appelle les «missions " ${ }^{7}$. Selon leur articulation avec les centres de pouvoir, les indigènes se sont vus impliqués dans les missions éducatives comme la mission Robinsón pour l'alphabétisation, la mission Ribas orientée vers l'acquisition rapide du titre de bachelier, et la mission Sucre qui ouvre des opportunités pour des études supérieures dans des systèmes parallèles d'Éducation supérieure. Ils ont reçu des soins d'attention primaire de la mission Barrio Adentro, de la formation pour le travail dans la mission Vuelvan Caras (aujourd'hui, mission Che Guevara), et ont bénéficié des centres de distribution d'aliments par le biais de la mission Alimentación. Dans toutes les missions du gouvernement, il y a des 
activités au sein des communautés indigènes. Il y a même une mission spécifique pour les populations indigènes dénommée la mission Guaicaipuro.

Plus récemment, les communautés se sont impliquées massivement dans la formation de " conseils communaux » (RBV 2006a) dans leurs villages respectifs. Ce type de structure donne le droit, avec seulement dix familles incorporées à un conseil, de proposer et d'exécuter un projet communautaire qui se monte pour la première année à 30 millions de bolivars (10 000 euros), projet qui, s'il réussit, peut permettre de formuler un autre projet la deuxième année, lequel recevra 60 millions de bolivars, voire davantage.

On peut dire que les communautés sont ainsi en train de s'incorporer de manière extrêmement accélérée à la gestion publique ${ }^{8}$. La politique d'inclusion implicite dans ce processus est aussi et avant tout une politique d'intégration au système étatique allant beaucoup plus loin que les anciennes stratégies qui se limitaient à faire cadeau de moteurs de bateau, de machettes et d'aliments et qui ne se préoccupaient pas de ce que les indigènes faisaient avec ces outils. La politique révolutionnaire est une stratégie agressive d'inclusion dans les services modernes (santé, éducation, sécurité et citoyenneté) orientés vers la lutte contre l'exclusion ainsiquela création et la stimulation de "l'hommenouveau », lequel, dans lecasdes autochtones, devrait être modelé idéalement selon ses modèles culturels.

Des sociétés indigènes qui, depuis plus de cinq siècles, ont cumulé les influences transformatrices du contact avec le monde extérieur et qui, depuis les années 1960, se sont habituées aux relations avec la société nationale et à l'incorporation d'éléments exogènes, ont établi les relations de dépendance nécessaires pour les conduire vers l'intégration à la société nationale. Des sociétés autonomes ont été incluses dans une unité majeure, l'État-nation, où elles ont tout de suite été transformées en sociétés marginales, dépendantes des réseaux de pouvoir de la société nationale. Les Indiens sont devenus des pauvres parmi les plus pauvres. Cela les a préparés à accompagner toute politique d'intégration/assimilation rapide à la société moderne, voire la révolution, dans le cadre des paramètres occidentaux de justice sociale, d'égalité et de lutte contre l'exclusion. L'arrivée de Chávez au pouvoir, avec laquelle le thème indigène prend de l'importance, ouvre pour l'État la possibilité d'accélérer la politique d'intégration des indigènes à la société nationale au nom des plus beaux idéaux sociaux.

Bien que l'intégration des sociétés indigènes à la société nationale ait été un rêve longuement caressé par les élites d'État, il n'y a jamais eu de consensus sur la manière d'y parvenir. Dès l'arrivée de Chávez au pouvoir, sa politique envers les indigènes a eu ses détracteurs, tant dans l'opposition que dans les rangs du mouvement chaviste. Durant les discussions sur la Constitution, le chapitre VIII sur les droits indigènes fut le seul à ne pas être approuvé en première discussion dans la chambre plénière de l'Assemblée nationale constituante. La polémique causée par la reconnaissance des territoires et par l'utilisation du terme " peuple » dans ce chapitre souleva d'énormes suspicions chez les militaires chavistes et fut 
amplement utilisée dans les medias par l'opposition politique (Mansutti Rodríguez 2000).

Par la suite, le thème indigène a perdu de sa pertinence pour l'opposition, mais gagné en notabilité pour la politique nationale et internationale du président. L'indigène a été extrêmement utile pour brandir des bannières qui appellent à l'alliance avec de puissants mouvements sociaux dans le monde, et spécialement en Amérique latine. Le thème de l'indigène fait partie intégrante de la politique internationale du président. Il ne voyage dans nul pays sud-américain sans la compagnie de dirigeants indigènes connus internationalement et, lorsqu'il arrive dans des pays comme la Bolivie et l'Équateur, il en profite pour se réunir avec des leaders amérindiens locaux, toujours du bord des dirigeants indigènes vénézuéliens qui l'accompagnent. Au niveau national, il leur octroie des postes importants. Noelí Pocaterra est parvenu à être la deuxième vice-présidente de l'Assemblée nationale, et elle fut nommée comme membre de la commission présidentielle pour la réforme de la Constitution. Pour sa part, Nicia Maldonado est la première ministre nommée au ministère pour les Peuples Indigènes, créé par Chávez en janvier 2007. En outre, elle fait partie, par disposition présidentielle, de la commission présidentielle pour l'expansion du pouvoir communal, le cinquième moteur de la révolution destiné à être la base sociale sur laquelle s'instaurera le modèle socialiste vénézuélien. Ce n'est pas tout. Les indigènes sont présents dans la publicité gouvernementale et sont inclus dans les événements nationaux et internationaux comme les foires du livre ou la foire internationale du tourisme de Caracas (Fitcar 2005, 2006). Lors de son allocution prononcée au moment de nommer les commissions présidentielles pour la réforme constitutionnelle et pour le pouvoir communal, le président, en parlant du socialisme, a appelé à prendre exemple sur les peuples indigènes et à suivre leur enseignement :

[...] Nuestros pueblos indigenas, a pesar de los siglos transcurridos, a pesar del bombardeo de antivalores, a pesar del atropello capitalista y del desmoronamiento de muchas de sus tradiciones, sin embargo han sido capaces, asi como resistieron a la agresión imperialista europea, han resistido también la agresión de los antivalores del capitalismo y en buena parte de sus espacios ellos conviven en socialismo originario, indigena. He dicho que muestro socialismo debe tener mucho de indoamericano, indovenezolano. Entonces, asi lo creo firmemente, debemos, debemos contribuir con el potenciamiento del poder comunal, del poder popular de nuestros pueblos indigenas, allá en su propio territorio, allá en sus comumidades, allá donde ellos conviven; porque ellos, a ellos les será mucho más fácil el tránsito al socialismo del siglo XXI [...]. (Chávez 2007a)

Les indigènes se sont convertis en image archaïque et originelle d'une révolution qui cherche son chemin. La société a beaucoup à apprendre d'eux afin de construire le nouveau socialisme. Nous pourrions être optimistes, si ce n'étaient les ombres au tableau du nouvel ordre politique. Les dispositions de la Constitution de 1999, de la résolution 169 de l'OIT et de la loi organique des peuples et communautés indigènes sont un blindage légal de premier ordre pour garantir les 
droits que la société nationale doit reconnaître aux peuples indigènes. Toutefois, il est facile de mettre en évidence que, lorsque cela touche aux points les plus stratégiques, la révolution fléchit. Or, ce qui est le plus stratégique pour les indigènes, c'est la reconnaissance pleine du droit à leurs territoires et à décider entre eux leur mode de vie. La disposition transitoire XII de la Constitution de 1999 établit que :

La demarcación del hábitat indigena a que se refiere el artículo 119 de esta Constitución, se realizará dentro del lapso de dos años contados a partir de la fecha de entrada en vigencia de esta Constitución. (RBV 2000)

Selon cette disposition, la démarcation devait être prête au plus tard le 15 mars 2002. À cette date, la loi de démarcation et de garantie des habitats et terres indigènes a seulement été promulguée. À ce jour, des titres de propriété collective ont été octroyés seulement à un très petit nombre de communautés kari'ña, warao, pumé et hiwi, et, encore, sur de faibles extensions de terres isolées les unes des autres et entourées de terres appartenant, soit à l'État, soit à des propriétaires privés. Les propositions de grandes extensions continues pour les peuples aux territoires les moins imbriqués avec des possesseurs de droits non indigènes ont été systématiquement bloquées, soit en leur mettant des conditions supplémentaires, soit en ne prenant pas de décision. Les cas des Barí de la Sierra de Perijá et des Ye'kwana-Sanema du Caura sont en ce sens emblématiques. Après que leurs documents ont été, non sans mal ${ }^{9}$, préalablement approuvés par la commission nationale de démarcation, y compris par les représentants militaires qui avaient jusque-là montré des résistances, ceux-ci ont été purement et simplement refusés au sommet de l'État.

$\mathrm{Si}$ on évalue le processus de démarcation, on rencontre de nombreuses faiblesses imputables à la politique du gouvernement ou, plutôt, à son absence de volonté politique. Cela inclut les rares ressources octroyées aux communautés pour promouvoir les propositions d'autodémarcation et pour faire fonctionner le pesant appareil bureaucratique créé selon les dispositions de la loi, et les erratiques directives de la Commission nationale envoyées aux commissions régionales. S'ajoute à cela que les représentants indigènes dans la commission nationale, huit au total, chacun d'entre eux représentant une région indigène, sont en grande majorité des dirigeants périphériques. Ils se trouvent, en conséquence, faiblement armés face au niveau d'expérience qu'exige une commission paritaire dans laquelle on compte des négociateurs de sept ministères ${ }^{10}$. Ces problèmes de structuration indigène au sein de la commission sont clairement en relation avec l'effet d'étirement-essoufflement provoqué par l'ouverture de postes politiques de haut niveau qui ont, en priorité, absorbé les meilleurs éléments du mouvement indigène vénézuélien.

Il est probable qu'à la source des problèmes que rencontre la démarcation se trouvent les appréhensions que réveillent dans l'État vénézuélien et chez ses 
militaires les demandes de territoires étendus par les peuples indigènes. Cette résistance n'est pas sans rappeler la teneur des discussions qui ont eu lieu sur ce thème à l'Assemblée nationale constituante. Un fragment du discours du Président prononcé au moment de remettre les premiers titres de propriétés de terre à des indigènes, livre un éclairage sans ambiguïté sur ce point :

[...] Ustedes tienen los documentos de propiedad de estas tierras, son de ustedes de hecho y de derecho, de tradición y de origen; soberania de los pueblos indigenas, por supuesto siempre enmarcado dentro de la soberanía nacional y la unidad territorial de la República; eso siempre hay que entenderlo, algunos hermanos indigenas tienen unas opiniones distintas a éstas, yo se las respeto por una parte, pero le decia a uno de ellos hace poco allá, en Miraflores ${ }^{11}$, yo le oía una exposición y le decia, yo no puedo estar de acuerdo contigo hermano, y no puedo estar de acuerdo contigo porque hay una Constitución y yo soy el Jefe del Estado; y yo estoy obligado a preservar la unidad Territorial de Venezuela, no podemos partir a Venezuela en varios pedazos, ;no! Eso sería traicionar la unidad nacional y traicionar el espíritu nacional; entonces en algumas commidades, algumos, yo creo que confundidos, han estado exigiendo lo que yo no puedo hacer, no me exijan lo que yo no puedo hacer, no me pidan la luna porque bueno se las regalo cuando esté allá llena en la noche, le digo allá está, pero no me pidan que la traiga aquí, no me pida nadie que yo le entregue título de propiedad sobre extensiones infinitas de territorio, sobre la mitad del estado Bolivar por ejemplo, no me pida nadie que yo le entregue los derechos que están en la Constitución y que son potestad del Estado, para explotar minas, para dar concesiones o para explotar petróleo, nadie, nadie se confunda; porque hasta ahí no vamos a llegar; primero que nada la unidad nacional, la unidad territorial, la unidad de un pueblo, que nadie se confunda o nadie se deje confundir: (Chávez Frias 2005, p. 16)

Il est très clair que, dans ce discours, le Président estime que les droits indigènes ont une limite qu'ils ne peuvent dépasser sans risquer de mettre en danger l'intégrité territoriale et la souveraineté que le peuple vénézuélien, en tant qu'unité supérieure et intégratrice des indigènes, possède sur la totalité du territoire national. Ces limites, dans la pratique et dans ce discours, sont associées à la superficie territoriale qui est sollicitée et au contrôle que l'un pourrait parvenir à avoir sur les ressources minérales qui sont octroyées à l'État par la Constitution. Le Président semble penser que le bon indigène est celui qui se satisfait, comme les paysans, d'une petite parcelle de terres et qui reconnaît qu'au-dessus de son droit à promouvoir une vie culturellement déterminée, il y a le droit de l'État, comme représentant de la volonté souveraine du peuple vénézuélien à «nous unifier ». Ce discours configurant la politique indigéniste du chavisme récupère toutes les peurs qui ont accompagné dans le monde la reconnaissance du droit de chaque peuple indigène à un territoire qui puisse être le fondement matériel et spirituel sur lequel il puisse promouvoir son droit à l'auto-détermination.

Un autre point que nous voulons souligner est la réticence dont a fait preuve le gouvernement chaviste à soumettre à l'approbation préalable des indigènes les macro-projets associés à son offensive politique internationale d'intégration. 
C'est le cas, par exemple, en ce qui concerne le gazoduc colombiano-vénézuélien de La Guajira, qui traverse des terres wayuu, et le grand gazoduc transamazonien, qui part de la côte nord du Venezuela, en traversant des terres kari'ña, pemón et akawaio, et arrive à Buenos Aires. Pour aucun d'entre eux, le gouvernement n'a réalisé la consultation qui est rendue obligatoire par la Constitution de 1999, la résolution 169 de l'OIT et la loi organique des peuples et communautés indigènes.

Force est de constater que les organisations indigènes, qui arrivent en 1999 à l'Assemblée nationale constituante solidement unies autour d'un programme propre, avec seulement un petit groupe d'opposants indigènes, sont aujourd'hui, huit années plus tard, bien fracturées. À partir de 2006, le CONIVE, l'unique organisation de niveau national, est divisé en deux grands courants, l'un mené par Noelí Pocaterra et le leadership traditionnel, et l'autre sous la houlette de la ministre récemment nommée Nicia Maldonado, tous deux comprenant des forces chavistes dirigeantes. Au niveau régional, la situation n'est guère meilleure. L'organisation régionale des peuples indigènes d'Amazonas (ORPIA) est aujourd'hui sérieusement confrontée à une nouvelle organisation, créée en 2005, promue et financée par le gouvernement régional d'Amazonas, appelée Confederación Indígena Bolivariana de Amazonas (COIBA). Le cas de l'Amazonas n'est pas une exception. En outre, toutes ces organisations subissent actuellement ${ }^{12}$ une forte pression afin qu'elles se dissolvent et que ses membres deviennent membres du parti socialiste unifié du Venezuela (PSUV) que promeut le gouvernement chaviste. De fait, les députés indigènes à l'Assemblée nationale, élus comme représentants du CONIVE et qui formaient un groupe politique autonome dans ce parlement, ont tous décidé de s'inscrire au PSUV et ont dissout leur groupe d'opinion au sein de l'Assemblée.

Si l'on doit faire un bilan, on peut voir que la politique révolutionnaire envers les indigènes permet de comprendre le niveau de soutien atteint par le chavisme parmi les électeurs indigènes, qu'on peut calculer aux environs de $80 \%{ }^{13}$, malgré les nuances à faire comme la maigre reconnaissance des terres et le déni du droit à la consultation préalable. En neuf années, la révolution bolivarienne a investi des ressources financières significatives au profit des communautés. Elle leur a apporté les missions éducatives et d'assistance. Elle a créé des bureaux pour la défense du peuple en charge de veiller sur leurs droits. Elle a donné de la présence politique internationale, nationale et régionale à ses dirigeants. Elle a nommé deux indigènes comme ministres et a confié à d'autres des fonctions de responsabilité. Elle a converti l'indigène en une icône et un archétype de la révolution au point, par moments, d'acquérir dans les discours des fonctionnaires du gouvernement des tons millénaristes. Si les organisations indigènes se maintiennent aux côtés de la révolution, c'est qu'elles ont de bonnes raisons pour cela. 


\section{« LA GÉOMÉtrie du POUVOIR 》: LES AMÉrINDIENS AUX PRISES AVEC LE SOCIALISME DU XXI ${ }^{\mathrm{c}}$ SIÈCLE}

Par leur profondeur et massivité, les changements promus par l'État dans le mode de vie des peuples indigènes correspondent aux transformations socioculturelles les plus fortes enregistrées depuis l'époque de l'exploitation du caoutchouc dans la Guayana, entre 1870 et 1923, ou celle de l'introduction de l'école (missionnaire ou laïque) dans les décennies 1960-1980. Le potentiel ethnocidaire des changements introduits par les nouvelles politiques ne doit pas être sous-estimé.

Ce que nous enseigne l'expérience indigène dans la révolution bolivarienne est que la reconnaissance légale des droits n'est pas nécessairement associée à son exercice effectif et peut, plutôt, finir par se convertir en une excuse pour galvaniser davantage les processus d'intégration et d'assimilation. La révolution s'est métamorphosée en un mécanisme homogénéisateur qui subordonne les intérêts propres des minorités ethniques aux objectifs stratégiques d'une révolution, promue par l'État, qui cherche à concentrer le pouvoir et à susciter des transformations radicales dans l'ensemble de la société nationale, y compris les peuples indigènes. À moyen terme, la révolution permet d'atteindre des objectifs d'Etat largement convoités : intégrer les indigènes au système national en sacrifiant sur l'autel de la révolution ce qui reste de leur autonomie sociale et politique. Le premier paradoxe est que la reconnaissance de droits qui demandent une intervention majeure de l'État - en l'absence de l'exercice d'une politique et d'un programme propre et autonome qui vise le développement de l'autodétermination sociétale et culturelle-, peut compromettre le droit le plus important, celui de maintenir des formes sociales alternatives autochtones. On améliore la qualité de certains services, on inclut, mais en même temps on met en doute la viabilité de la différence sociale et culturelle.

Dans tous les cas, la justice et la pertinence des droits légaux ne doivent pas être jugées par la qualité de leur rédaction, mais par celle de leur application. La Constitution bolivarienne établit clairement dans l'article 119 que l'État reconnaît aux peuples aborigènes leur droit originel de leurs habitats et terres, tandis que l'article 121 reconnaît leur droit de décider de leurs propres modes de vie ${ }^{14}$. Les deux articles créent les conditions pour donner aux indigènes le droit de maintenir leurs cultures à l'intérieur d'espaces protégés, sans autre pression que l'évolution même de leurs sociétés. Ce sont les objectifs par excellence qui donnent du sens à la politique indigène. Si les droits reconnus dans ces articles n'ont pu se réaliser pleinement, cela ne provient pas des textes, mais des priorités établies au sein du processus révolutionnaire auquel les dirigeants autochtones ont participé. Lorsque l'exercice des droits est hiérarchisé et filtré par des agents de l'État et que ce sont eux qui décident des priorités, cela entraîne inéluctable- 
ment que l'on place en premier celles qui sont acceptables et plus faciles à mettre en œuvre pour le monde majoritaire créole, et pas nécessairement celles qui ont une importance déterminante pour les indigènes. Ainsi, par exemple, ce n'est pas la même chose de recevoir des fonctionnaires qui doivent développer des missions éducatives ou de santé dans un territoire indigène sans titre de propriété et sans gouvernement local reconnu par l'État, que de recevoir ces mêmes fonctionnaires dans un territoire reconnu où ces habitants indigènes exercent leur gouvernement et administrent leur territoire de façon à garantir leur propre mode de vie. Le contrôle social de l'espace propre aux indigènes doit donner du sens aux politiques étatiques qui se réalisent sur place, et pas le contraire.

On observera que l'exercice d'autres droits, différents des droits territoriaux mais également reconnus par la Constitution, a été privilégié comme, par exemple, la participation politique, la juste rémunération du travail, la promotion du développement d'entreprises et d'activités économiques et l'éducation scolaire et professionnelle. Or il s'agit de droits, certes importants, mais qui réfèrent à des relations renforçant la dépendance des peuples aborigènes vis-à-vis de la société nationale. Tous signifient l'inclusion dans le jeu de l'État-nation.

Les résultats obtenus avec l'exercice de ces droits contredisent l'esprit de l'article 121 de la Constitution qui est au cœur des droits des indigènes. Sans une politique propre de renforcement culturel, les indigènes sont en train de contribuer à incorporer dans leurs groupes de nouvelles pratiques culturelles qui affectent les dynamiques de changement en affaiblissant les valeurs fondamentales de leur propre mode de vie, cela en intervenant comme législateurs et fonctionnaires et en demandant des crédits, en créant des coopératives et des conseils communaux, en se formant dans des écoles professionnelles ou des universités.

Plusieurs problèmes rencontrés sont aussi reliés à la nature du leadership indigène et de ses intérêts. La participation politique active dans la société nationale requiert que le représentant connaisse et manie tant le langage de cette pratique que ses habitudes et protocoles. Cela ne s'apprend qu'en ayant eu des expériences interculturelles significatives avec des acteurs du monde créole, comme la mission ou l'école, le militantisme politique et la ville. Les dirigeants principaux sont, par conséquent, des leaders à expérience urbaine qui ont déjà goûté au miel de la société nationale et qui vont évaluer le stratégique des objectifs de leur peuple à partir de leur expérience.

Dans les communautés, la situation tend à se reproduire. Les interlocuteurs localement sont en général ceux qui sont passés par les écoles ou les missions et qui ont eu des expériences et des relations avec les institutions du monde extérieur (Alès 2006, pp. 158-160). Ils rivalisent avec les leaders traditionnels et ont fréquemment des postes publics, certains temporaires et politiques comme les charges de promoteur des mairies et du gouvernement régional, d'autres permanents comme les maîtres et les infirmiers. Dans certains cas, les deux peuvent se combiner. 
Avec des représentants politiques nationaux, régionaux et locaux, qui ont pour paradigme que la manière dont ils s'intègrent eux-mêmes à la société nationale est la meilleure, il semble bien difficile que s'élaborent des stratégies interculturelles dans lesquelles les expectatives des indigènes moins créolisés se voient représentées. Le mode de vie des communautés et leurs propres aspirations restent diffus et difficilement visibles pour ceux qui sont chargés en principe de les défendre. C'est bien là le paradoxe entre représentants et représentés. Et la négociation interculturelle est laissée de côté.

Dans une situation politique nationale caractérisée par la massivité des actions et la célérité des changements que promeut un gouvernement qui cherche à consolider les conditions lui permettant de poursuivre la construction du socialisme dans le pays, les organisations indigènes sont doublement désavantagées. D'une part, leurs représentants les plus fameux, dotés d'une plus grande expérience interculturelle, ont laissé de côté des objectifs stratégiques du programme défendu lors de la Constituante et ont eu des difficultés pour adapter ce même agenda aux nouvelles circonstances révolutionnaires. De l'autre, la révolution avance rapidement dans l'implantation de modèles propres, d'un « homme nouveau », ajusté aux intérêts de la révolution, qui ne sont pas nécessairement ceux des communautés.

Toute révolution doit construire une hégémonie qui la maintienne. Dans sa phase initiale, la construction de cette hégémonie implique d'imposer de nouveaux points de référence et de nouvelles manières de faire et de penser. Si les acteurs politiques qui appuient la révolution ne peuvent pas défendre avec force la valeur de leurs expectatives comme groupe social au sein du processus révolutionnaire, ils courent le risque de se voir vaincus par la force des intérêts des groupes qui, eux, en sont capables et qui leur donnent forme dans les pratiques de la révolution et de ses idéologies. Le leadership indigène, dans ce cas, brille davantage par ses discours révolutionnaires que par ses prises de position sur le programme proprement indigène, qui se trouve ainsi subordonné à la rhétorique du programme révolutionnaire national.

En bâtissant son hégémonie, la révolution vénézuélienne a créé en même temps les conditions pour contrôler la fidélité des révolutionnaires. Dans une révolution ayant pour références l'antagonisme de classes dans le capitalisme et la lutte contre l'impérialisme, tout ce qui n'est pas conforme aux intérêts de la révolution est immédiatement qualifié de contre-révolutionnaire. Dans ces circonstances, les leaders de la révolution doivent veiller constamment à ne pas contrarier les desseins du gouvernement.

Exercer des pressions sur le gouvernement afin qu'il réalise le programme indigène a un résultat compromettant pour tout leader s'il n'a pas, auparavant, obtenue la garantie que le gouvernement est d'accord. Cela explique le silence public des dirigeants indigènes dans les dossiers de la démarcation des terres et de la non-exécution de la consultation préalable dans les projets de l'État qui 
peuvent affecter les populations indigènes. Ils ont préféré faire du lobbying dans les hautes instances que d'affronter publiquement le gouvernement comme ils le faisaient avant l'arrivée de Chávez à la tête du pays.

Un autre élément fondamental explique la situation. En s'articulant activement au processus révolutionnaire et en prétendant grimper dans sa structure dirigeante, les représentants amérindiens doivent jouer le jeu de la politique, conçue individuellement comme un chemin qui permet de parvenir aux plus hautes positions de la hiérarchie. Il est nécessaire de bien prendre en compte toutes les dimensions de cette ambition. En cherchant à faire carrière dans la politique régionale, nationale et internationale, ce que veulent avant tout montrer les autochtones, c'est l'antithèse de l'indigène " d'en bas ». Cette combinaison de circonstances les amène à donner une plus grande importance à leur carrière politique, pour terminer par faire le jeu du système politique étatique.

Dans des sociétés en mutation, où un secteur révolutionnaire prend le pouvoir de l'État et cherche à imposer son hégémonie, les conflits ne sont pas administrés comme dans les sociétés libérales conventionnelles où les différences idéologiques au sein du groupe majoritaire sont davantage tolérées. En suscitant un conflit, ou simplement en faisant part d'une différence d'opinion, ses promoteurs révolutionnaires doivent mesurer avec précision, sous peine de tomber en disgrâce, en quoi cela profite à l'État et à ses dirigeants. Dans la révolution bolivarienne, les représentants amérindiens suscitent fort peu de conflits et, quand ils le font, cela se cantonne au cadre conventionnel imposé par la révolution. Il est essentiel de considérer que cette attitude est cohérente avec le fait qu'ils se sentent importants pour la première fois dans un projet de construction nationale. C'est pourquoi ils ne sont pas disposés à mettre en péril ce privilège obtenu après tant d'années d'humiliations, de racisme et de mépris de la part de la société nationale.

Les expériences des peuples autochtones vivant dans des États socialistes totalitaires n'ont pas été, jusqu'à présent, particulièrement positives. En général, la conception prédominante a été la même que dans des États considérés comme non totalitaires : dans tous les projets où des sociétés sont soumises à un État centralisateur, les uns et les autres cherchent à imposer des modèles homogénéisateurs et hégémoniques où la différence culturelle n'est concevable qu'une fois subordonnée et réduite à des aménagements politico-administratifs mineurs et à une forme de folklore. Dans ces circonstances, les combats à mener dans l'un ou l'autre modèle d'État exigent des peuples autochtones ayant des projets et des objectifs clairs, qu'ils soient capables de négocier à l'intérieur de la société les meilleures conditions pour la survie de leurs cultures. Si l'on considère l'espoir incarné par les indigènes élus ou nommés, cela n'est pas en train de se produire dans la phase présente de la révolution vénézuélienne.

Ce n'est qu'au prix de la contestation, de la discussion, de la mise en cause, en d'autres termes, de la critique que la démocratie peut se maintenir. La contestation ne venant pas des élus, elle ne peut venir que des organisations de base. 
C'est ce qu'elles commencent timidement à faire, au grand dam des députés indigènes et des ministres nommés. Ici, elles protestent contre les refus de démarcation des territoires indigènes, là, contre l'absence de consultation pour des projets concernant des territoires autochtones ou, encore, contre les projets de réformes des lois indigènes et l'instauration de " territoires communaux » qui ne leurrent personne par rapport à ce que signifie une véritable démarcation et l'octroi de titres de propriété.

On peut donc réaffirmer que le rapport des peuples indiens avec les sociétés étatiques modernes où ils sont englobés est toujours difficile, tout État étant porteur d'un idéal civilisateur avec un modèle de citoyen qu'il essaie d'imposer à tous ses ressortissants, même s'il s'agit d'un État révolutionnaire agissant au nom des indigènes. Dans ces relations, toujours asymétriques, les sociétés indigènes qui sont le moins soumises à la logique de la société nationale ont les meilleures possibilités de survivre culturellement de façon différenciée. Ce que nous enseigne l'histoire, c'est que les sociétés indigènes qui ont le mieux surmonté leurs relations avec les sociétés étatiques modernes ont été celles qui avaient créé des relations visant à garantir un degré notable d'autonomie politique, économique et sociale.

Mais que faire quand une révolution prend place au nom des indigènes et quand ces derniers sont sollicités pour devenir des protagonistes? Ce que nous montre la situation vénézuélienne, c'est que si les indigènes s'approprient politiquement l'État et font le jeu de sa logique, ils sacrifient alors leurs intérêts stratégiques au nom des intérêts de l'État révolutionnaire. Cela fait, on est alors au bord du suicide. Sortir leur épingle du jeu demande aux indigènes de faire ressortir la valeur de leur différence culturelle et de récupérer leurs objectifs stratégiques initiaux pour les faire valoir au sein de la révolution. Si cela arrivait, la vocation hégémonique de la révolution serait freinée et le mouvement indigène aurait davantage la possibilité de faire valoir son droit à l'autonomie et à la reproduction sociale. On pourrait ainsi arriver à une véritable révolution : celle où les indigènes dans un État moderne auraient non seulement le droit, mais également la possibilité de se reproduire socioculturellement tels qu'ils sont. La révolution en sera une vraiment lorsqu'être révolutionnaire sera pour les leaders indigènes simplement être Indien.

Alexander Mansutti Rodriguez CIAG/UNEG, Ciudad Bolivar

Catherine Alès CNRS, Paris 


\section{NOTES}

1. Jusqu'à l'entrée en vigueur de la nouvelle constitution, on parlait au Venezuela de "Fuerzas Armadas » composées par quatre éléments autonomes: l'armée, la marine, l'aviation et la garde nationale. Elles sont désormais la " Fuerza Armada » et sous le contrôle d'un seul commandement, le chef de l'État.

2. Le principe du " protagonisme » renvoie, théoriquement, à la démocratie directe et, pratiquement, au fait que les communautés élaborent et dirigent, sans intermédiaires, les projets à même de résoudre leurs problèmes.

3. Étant donné le succès des conseils communaux, aujourd'hui répandus dans tout le pays, le gouvernement a décidé de fonder ce qu'il dénomme la « nouvelle géométrie du pouvoir ». L'expansion et la consolidation du pouvoir communal dans tout le pays sont considérées comme l'objectif principal de cette étape de la révolution. Il est estimé comme étant le cinquième moteur de la construction socialiste et la structure à partir de laquelle le programme de formation politique et la nouvelle division politico-territoriale du nouvel État doivent s'élaborer. En ce moment, la loi qui régule les conseils communaux est en train d'être réformée afin de leur donner les compétences que leur place novatrice au sein du projet stratégique de l'État exige.

4. Effectivement, l'expérience interculturelle et les capacités d'interrelations accumulées par chaque peuple amérindien, y compris pour des secteurs différenciés au sein de chacun d'entre eux, sont variables et déterminent les modalités de relations que ces groupes et communautés établissent avec l'État et avec d'autres acteurs sociaux non indigènes.

5. Cet article établit que les dispositions sur les droits de l'homme contenues dans des accords internationaux approuvés par l'Assemblée nationale, tels que la résolution 169 de l'OIT, sont supérieures à toutes les dispositions des lois organiques et ordinaires vénézuéliennes, y compris, de la propre constitution lorsque leurs normes sur la jouissance et l'exercice des droits de l'homme favorisent davantage le citoyen.

6. Cette loi n'est restée en vigueur que quelques mois. Une fois que le gouvernement décide de centrer sa stratégie de gestion publique sur les conseils communaux, plusieurs de ses dispositions entrent en contradiction avec la stratégie de consolidation de la révolution ; la loi est alors abrogée très rapidement.

7. Les « missions » sont des projets d'attention parallèles aux institutions de l'État destinées à régler tout problème social. Les personnes y travaillent comme des promoteurs volontaires qui ne reçoivent pas de salaires si ce n'est des « bourses » ou des « aides », et ne reçoivent pas non plus d'ordre des institutions formelles de l'État si ce n'est de chefs nommés ad hoc par la présidence de la République (MPPCI 2007). Lorsque ces projets se consolident, ils constituent des réseaux de prestation de services. La réforme de la Constitution de 1999 en cours (Chávez 2007b) confère aux missions un rang constitutionnel; elles seront, si la reforme est approuvée, au même niveau que l'administration publique conventionnelle.

8. Dans certaines communautés, les indigènes ont déjà plusieurs conseils communaux, y compris, dans des communautés éloignées des centres urbains.

9. Plusieurs réquisits supplémentaires par rapport au cadre de la Loi de démarcation ont été réclamés à la dernière minute aux indigènes qui essayaient de monter des propositions d'autodémarcation, comme des recensements de la population (sans crédits alloués pour ce faire) et des ethnographies complexes, retardant ainsi toujours davantage l'exercice.

10. Le Ministerio del Ambiente, le Ministerio de la Defensa, le Ministerio de Energía y Minas, le Ministerio de Educación, le Ministerio de Producción y Comercio, le Ministerio de Relaciones Exteriores et le Ministerio de Relaciones Interiores.

11. Palais présidentiel du Venezuela, situé dans le centre de Caracas.

12. Avril-septembre 2007.

13. Ce chiffre est une estimation minimale effectuée à partir des résultats des élections présidentielles de 2006 dans les municipalités dont la population est majoritairement indigène (CNE 2007). 
14. Article 121 : "Los pueblos indigenas tienen derecho a mantener y desarrollar su identidad étnica y cultural, cosmovisión, valores, espiritualidad y sus lugares sagrados y de culto. El Estado fomentará la valoración y difusión de las manifestaciones culturales de los pueblos indigenas, los cuales tienen derecho a una educación propia y a un régimen educativo de carácter intercultural y bilingüe, atendiendo sus particularidades socioculturales, valores y tradiciones » (RBV 2000).

\section{RÉFÉRENCES CITÉES}

ALÈs Catherine

2003 «Función simbólica y organización social. Dircursos rituales y política entre los yanomami ", in Catherine Alès et Jean Chiappino (éds), Caminos cruzados. Ensayos en antropología social, etnoecología y etnoeducación, IRD Éditions/ ULA-GRIAL, Mérida, pp. 197-240.

2006 Yanomami, l'ire et le désir, Éditions Karthala, Paris.

2007 «"Le goût de miel": le nouvel ordre politique dans l'Amazonie vénézuélienne et la participation yanomami », Journal de la Société des Américanistes, 93 (1), pp. 227-255.

Chávez Frias Hugo

2005 Entrega de títulos colectivos de tierras y hábitat indígenas desde Santa Rosa de Tácata, Municipio Freitas, Estado Anzoátegui, 09 de agosto de 2005, www.gobiernoenlinea.ve/misc_view/ver_alocución.Pag?fecha_buscar.200508-09 (09/06/2006)

2007a Juramentación del Consejo Presidencial para la Reforma Constitucional y del Consejo Presidencial del Poder Comunal, www.mci.gov.ve/doc/17ene2007 juramentacion.doc (03/05/2007)

2007b Anteproyecto para la 1a reforma constitucional. Propuesta del Presidente Hugo Chávez, agosto 2007, www.gobiernoenlinea.gob.ve/noticias-view/share File/Proyecto.pdf (10/09/2007)

CNE

2007 Consejo Nacional Electoral, www.cne.gob.ve (09/01/2007)

Fitcar

2005 http://www.mipunto.com/temas/4to_trimestre05/fitcar_2005.html

2006 http://www.fitcar.com.ve/ (03/05/2007)

Mansutti RodRíguez, Alexander

1992 "Una mirada al futuro de los indígenas de Guayana », Boletín Antropológico, 29 , pp. 7-27

2000 "Pueblos indígenas y asamblea constituyente en Venezuela ", Revue internationale des Droits des Peuples Autochtones, 2, pp. 35-49.

s. d. "La demarcación de territorios indigenas en Venezuela : algunas condiciones de funcionamiento y el rol de los antropólogos », Antropológica [sous presse].

Ministerio del Poder Popular para la Comunicación e Información (MPPCI)

2007 Misiones Bolivarianas, http://www.misionesbolivarianas.gob.ve/(03/05/2007). 


\section{República Bolivariana de Venezuela (RBV)}

2000 "Constitución de la República bolivariana de Venezuela », Gaceta Oficial, $\mathrm{n}^{\circ} 5.453$ de la República bolivariana de Venezuela, 24 de marzo, Caracas.

2001a "Ley aprobatoria del Convenio n 169 sobre Pueblos Indigenas y Tribales », Gaceta Oficial, $\mathrm{n}^{\circ} 37.305,17$ de octubre, Caracas.

2001 « «ey de Demarcación y Garantía de los Hábitat y Tierras Indígenas », Gaceta Oficial, $\mathrm{n}^{\circ} 37.118,12$ de enero, Caracas.

2002 «Ley de Diversidad Biológica », Gaceta Oficial, n 5.468, extraordinaria del 24 de mayo, Caracas.

2005a «Ley Orgánica de Pueblos y Comunidades Indígenas », Gaceta Oficial, $\mathrm{n}^{\circ} 38.344,27$ de diciembre, Caracas.

2005b "Ley Orgánica del Poder Público Municipal », Gaceta Oficial, n 38.204, 08 de junio, Caracas.

2006a "Ley de los Consejos Comunales », Gaceta Oficial, n 5.806, extraordinaria del 10 de abril, Caracas.

2006b « Ley Orgánica para la Gestión, Planificación y Ordenamiento del Territorio, Caracas », Gaceta Oficial, n 38.388, 01 de marzo, Caracas.

s. d. " Derechos históricos de los pueblos indígenas. Avances y logros legislativos », Asamblea Nacional de la República Bolivariana de Venezuela, Caracas (tríptico). 